\section{Diabetes and hypertension are possible long-term effects of shock-wave lithotripsy}

Shock-wave lithotripsy (SWL) has been used in the minimally invasive treatment of renal and proximal ureteral stones for over 20 years. The transient, acute effects of SWL on renal and other tissues are well known, but studies investigating medium-term effects have reported conflicting results. As long-term data are limited, Krambeck et al. carried out a 19-year follow-up of 630 patients who had been referred to the Mayo Clinic in 1985 for SWL treatment of renal or ureteral stones.

Of 578 patients alive in 2004, 288 completed a questionnaire focusing on possible adverse medical effects of SWL. Control patients (with conservatively treated urolithiasis) were matched for age, sex, and year of presentation.

Overall, SWL-treated patients were 1.5 times more likely to develop hypertension than controls; this risk was highest for patients given bilateral SWL. SWL-treated patients were also over three times more likely to develop diabetes than controls, an association that was independent of BMI. The risk of developing diabetes was related to the average intensity and number of shocks administered. Laterality of treatment did not affect this risk, however, possibly because part of the pancreas lies in the lithotripsy blast path regardless of the side of the body being treated.

Interestingly, although diabetes risk was independent of BMI, obese SWL-treated patients were more likely than lean SWLtreated patients to develop diabetes, which suggests that the presence of pre-existing risk factors for this condition might increase the risk of developing diabetes post-SWL.

Original article Krambeck AE et al. (2006) Diabetes mellitus and hypertension associated with shock wave lithotripsy of renal and proximal ureteral stones at 19 years of followup. J Urol 175: 1742-1747

\section{Paternal age over 40 years linked with reduced reproductive success}

Maternal age $>35$ years has long been known to be an adverse risk factor for reproductive outcomes. Paternal age, on the other hand, has only recently been acknowledged to have an effect on reproductive outcomes, although the effects of aging on male sexual activity and on failure to conceive, respectively, have been hard to distinguish. A new study has now confirmed that paternal age $>40$ years is an independent risk factor for failure to conceive.

The authors analyzed data from 59 French centers offering in vitro fertilization (IVF). A total of 1,938 couples were identified, who were seeking IVF treatment because the female partner had bilateral tubal obstruction (or absence). The risk of IVF failure was calculated for men and women in each 5-year age-group (the 35-40 year agegroup in women was further subdivided at age 37 years, because of the importance of this threshold in published literature).

The risk of IVF failure increased with advancing age for both men and women; in women, risk was substantially increased for those aged $\geq 38$ years, confirming the results of previous studies. For men, the risk of IVF failure approximately doubled in those aged $\geq 40$, and was especially high in men whose partners were aged $\geq 41$ years (odds ratio $5.74,95 \%$ $\mathrm{Cl}$ 2.16-15.23). Some age-groups comprised few couples, but when analysis was limited to age-groups containing at least 30 couples, this association persisted. Paternal age $>40$ years was associated with an increased risk of IVF failure, especially when the woman was aged $\geq 35$ years.

Mechanisms for the effect of paternal age on reproductive success remain to be determined.

Original article de La Rochebrochard E et al. (2006) Fathers over 40 and increased failure to conceive: the lessons of in vitro fertilization in France. Fertil Steril 85: 1420-1424

\section{Preoperative renal core biopsy for incidental tumors}

Performing preoperative renal core biopsy of incidentally detected renal masses could avoid unnecessary surgery in up to a third of cases, according to a recent Australian study.

The widespread use of radiologic imaging in recent years has led to an increase in the incidental detection of small renal masses. These masses are usually treated with surgical resection, even though some lesions turn out to be benign. In order to determine the incidence of benign lesions among incidentally detected masses, Vasudevan and colleagues 